\title{
Desafios para o aprendizado de neurorradiologia na graduação médica: análise do ponto de vista discente
}

\section{Challenges for learning neuroradiology in undergraduate medical school: analysis from the students' point of view}

\author{
Esther de Alencar Araripe Falcão Feitosa ${ }^{1,2}$ (D) estherfalcaofeitosa@gmail.com \\ Luiz Henrique Costa Neto² (D) luizh_medicina@edu.unifor.br \\ Carina de Oliveira Gregório ${ }^{1}$ (1) carina_og09@outlook.com \\ Letícia Nobre Limas' ${ }^{1}$ (D) leticialimas97@outlook.com \\ Paulo Goberlânio de Barros Silva' (1) paulo_goberlanio@yahoo.com.br \\ Carlos Eduardo Barros Jucá1,2 (1) | eduardojuca@gmail.com
}

\begin{abstract}
RESUMO
Introdução: O ensino da neurorradiologia na graduação médica deve ser encarado como uma ferramenta de integração para o estudo interdisciplinar de radiologia, anatomia e neurologia. Na prática, percebe-se uma limitação dos alunos em adquirir tais conhecimentos, seja pela"neurofobia", seja pela falta de conhecimentos anatômico-radiológicos prévios e também pela carência de materiais didáticos integrados direcionados para a graduação. Contudo, há poucos trabalhos relatando as dificuldades encontradas pelos alunos no aprendizado de neurorradiologia.

Objetivo: Avaliar a percepção dos estudantes de medicina sobre as dificuldades do aprendizado em neurorradiologia.

Método: Estudo quantitativo, realizado com estudantes de medicina matriculados no segundo e no sétimo semestres de uma universidade de Fortaleza. Os dados foram obtidos por meio de questionário estruturado com 12 questões de respostas sim ou não. As questões versaram sobre as possíveis dificuldades encontradas para aquisição do conhecimento neurorradiológico, dentre elas: à carência de material didático direcionado, à falta de integração com a neurologia, à necessidade de conhecimentos básicos radiológicos e anatômicos, ao volume de assunto, e às limitações das metodologias ativas e tradicionais.

Resultados: Foram analisados 181 questionários. Grande parte discente refere como dificuldade a necessidade de ter conhecimentos básicos prévios de radiologia (80,1\%); de neuroanatomia (77,5\%); e de correlacionar radiologia e neuroanatomia (70,9\%). Quando comparados os grupos do 20 semestre e do 70 semestre, houve uma tendência maior a apontar a ausência de conhecimento prático de neurologia pelos alunos do 20 semestre como um fator de maior dificuldade para o aprendizado de neurorradiologia ( $82,6 \%$ versus $67,4 \%$, com $p<0,0018)$. Quando perguntados sobre a utilidade da criação de um e-Book direcionado para a graduação para o aprendizado de neurorradiologia, 85,6\% dos alunos responderam afirmativamente; no caso de um aplicativo, 92,3\% concordaram. Quanto à correlação entre a neurorradiologia e a prática médica, 98,3\% responderam que é um conhecimento útil e necessário.

Conclusão: Na opinião dos estudantes, os conhecimentos prévios de neuroanatomia e de neurologia clínica são importantes para o aprendizado de neurorradiologia. A elaboração de um material como e-book ou aplicativo com foco em integrar o ensino dessas disciplinas é considerada uma
\end{abstract} boa alternativa para facilitar a compreensão da neurorradiologia.

Palavras-chave: Educação Médica; Radiologia; Neurologia; Neurociências; Ensino; Material Didático; Estudantes de Medicina; Neurofobia.

\section{ABSTRACT}

Introduction: The teaching of neuroradiology in undergraduate medical school must be seen as an integration tool for the interdisciplinary study of radiology, anatomy and neurology. In practice, there is a limitation on the part of students in acquiring such knowledge, either due to "neurophobia", or due to the lack of previous anatomical-radiological knowledge and also due to the lack of integrated didactic materials aimed at undergraduate school. However, there are few studies reporting the difficulties encountered by students in learning neuroradiology.

Objective: To assess the perception of medical students about learning difficulties in neuroradiology.

Method: Quantitative study, carried out with medical students enrolled in the second and seventh semesters of a university in Fortaleza. Data were obtained through a structured questionnaire with 12 yes or no answer questions. The questions addressed the possible difficulties encountered in acquiring neuroradiological knowledge, among them: the lack of targeted didactic material, the lack of integration with neurology, the need for basic radiological and anatomical knowledge, the large volume of content to study, and the limitations of active and traditional methodologies.

Results: 181 questionnaires were analyzed. Most students report as difficulties: the need for basic knowledge of radiology (80.1\%); neuroanatomy (77.5\%); and to correlate radiology and neuroanatomy (70.9\%). When comparing the 2nd semester and 7th semester groups, there was a greater tendency to point out the lack of practical knowledge of neurology by 2 nd-semester students as a factor of greater difficulty in learning neuroradiology (82.6\% versus $67.4 \%$, with $p<0.0018$ ). When asked about the usefulness of creating an e-book aimed at undergraduate students for learning neuroradiology, $85.6 \%$ of the students answered affirmatively; in the case of a mobile application, 92.3\% agreed. As for the correlation between neuroradiology and medical practice, $98.3 \%$ answered that it is useful and necessary knowledge.

Conclusion: In the students' opinion, previous knowledge of neuroanatomy and clinical neurology is important for learning neuroradiology. The development of material such as an e-book or mobile application focused on integrating the teaching of these disciplines is considered a good alternative to facilitate the understanding of neuroradiology.

Keywords: Medical Education; Medical Student; Medical School; Radiology; Neurology; Neuroscience.

${ }^{1}$ Centro Universitário Christus, Fortaleza, Ceará, Brasil.

2 Universidade de Fortaleza, Fortaleza, Ceará, Brasil.

Editora-chefe: Daniela Chiesa

| Editor associado: Pedro Tadao Hamamoto Filho

Recebido em 04/14/20; Aceito em 12/05/20.

Avaliado pelo processo de double blind review. 


\section{INTRODUÇÃO}

O ensino da neurorradiologia para a graduação médica pode funcionar como uma ferramenta de integração interdisciplinar e transdisciplinar dentro da matriz curricular das universidades, introduzindo o desenvolvimento de competências assistenciais desde o início de sua abordagem no curso médico ${ }^{1,2}$. A inserção precoce da radiologia nos primeiros semestres do curso tem efeito positivo, visto que essa área permite a elucidação de processos fisiopatológicos e alterações morfofuncionais ainda dentro de semestres básicos do curso de Medicina ${ }^{1-6}$.

Uma grande limitação apontada pelos estudantes para a fixação dos conhecimentos das áreas básicas é justamente a falta de integração com a prática médica ${ }^{2}$ A falta de conhecimento sobre as possíveis aplicações práticas do que está sendo estudado gera desinteresse ${ }^{7-9}$. A radiologia, neste contexto, pode auxiliar na introdução de conceitos clínicos de forma introdutória dentro do curso, fazendo elo com questões práticas do cotidiano médico ${ }^{10}$.

É cada vez mais comum nos cursos de Medicina a mudança da matriz curricular com o intuito de adotar as metodologias ativas como pilares do ensino, permitindo que o aluno busque livremente o conteúdo necessário, emponderando-o do seu próprio conhecimento ${ }^{11,12}$. Para o sucesso do método, porém, é necessário que o discente possua intimidade e confiança com as diversas fontes bibliográficas disponíveis. Além de artigos científicos e livros, atualmente é possível encontrar e-books, sites, aplicativos e até mesmo redes sociais. Contudo, essas últimas fontes menos tradicionais, embora sejam muitas vezes até mais didáticas, costumam gerar dúvidas quanto à confiabilidade, limitando de certo modo o seu uso de forma mais ampliada ${ }^{2}$

Na prática docente, a neurorradiologia é considerada um assunto complexo pelos alunos. Alguns fatores podem ser implicados, como a neurofobia relacionada às neurociências $s^{8,9,13}$; a limitação na compreensão dos aspectos mais básicos da radiologia em si, como a física aplicada à Medicina e o uso correto das terminologias radiológicas ${ }^{2}$; além da noção de tridimensionalidade dos aspectos anatômicos. Percebe-se ainda carência de material direcionado para a graduação, limitando o aprendizado na ausência de um profissional radiologista como guia, algo que vai de encontro às bases do aprendizado por meio de metodologias ativas ${ }^{2}$.

Embora muito seja proposto, há poucos trabalhos nacionais direcionados para esta avaliação, limitando a viabilização de soluções para os possíveis problemas. Sendo assim, é fundamental avaliar a opinião dos discentes sobre as dificuldades encontradas para o aprendizado de neurorradiologia, com o intuito de subsidiar a aquisição de conhecimentos neste campo ${ }^{14,15}$.
O objetivo deste trabalho foi analisar a percepção dos estudantes de Medicina sobre os desafios no ensino da neurorradiologia, inclusive avaliando o uso das metodologias ativas nesse processo, permitindo a construção de estratégias que estimulem o aprendizado.

\section{MÉTODOS}

Trata-se de umestudotransversal equantitativo, realizado por meio da elaboração de um questionário estruturado direcionado a alunos de uma faculdade de Medicina privada situada na cidade de Fortaleza (CE), que utiliza estratégia de ensino centrada no aprendizado baseado em problemas (ABP), a qual apresenta ferramentas pedagógicas que estimulam o aluno a buscar o conhecimento, diferentemente da metodologia passiva, composta predominantemente por aulas expositivas.

O questionário possuía um total de 16 itens e foi direcionado para avaliação das dificuldades enfrentadas pelos discentes no estudo de neurorradiologia. As perguntas versaram sobre características demográficas da população e suas concepções pessoais sobre o ensino de neurorradiologia na graduação.

Os critérios de inclusão foram que os alunos estivessem matriculados e com assiduidade maior que $75 \%$ nos semestres com neurorradiologia: o segundo (devido ao módulo de sistema nervoso central) e o sétimo semestre (neurologia clínica) do curso de graduação em Medicina. Foi considerado como único motivo de exclusão a ausência deste critério.

Para a análise e disposição dos resultados, foram utilizados os softwares Epi Info 7 (CDC, 2019) e Google Forms. Os resultados numéricos foram expressos em forma de frequência absoluta e percentual e analisados pelos testes exato de Fisher e qui-quadrado de Pearson.

Quanto aos aspectos éticos, todos os respondentes decidiram livremente sobre a participação neste estudo, assinando o Termo de Consentimento Livre Esclarecido e recebendo uma via do documento. Este estudo foi aprovado pelo Comitê de Ética em Pesquisa do Centro Universitário Christus, sob o parecer consubstanciado número 3.021.673. Não houve conflito de interesse por parte dos pesquisadores.

\section{RESULTADOS}

Ao todo, foram analisados 181 questionários, sendo 91 alunos do $2^{\circ}$ semestre da faculdade de Medicina, em contato com disciplinas básicas, como neurociências e neuroanatomia; e 90 alunos do 70 semestre, cursando módulos clínicos, como neurologia, neurocirurgia e trauma. Todos os questionários foram aproveitados, não sendo necessário excluir nenhum discente. A maioria dos alunos tinha mais que 20 anos (67.6\%) e houve um leve predomínio de mulheres (58,6\%). A maioria $(65,2 \%)$ possuía veículo próprio. 
Inicialmente foram feitas cinco perguntas para avaliar fatores que pudessem contribuir para a dificuldade no ensino da graduação de neurorradiologia. As duas primeiras buscaram investigar se a necessidade de ter conhecimentos básicos sobre radiologia e neuroanatomia poderia representar uma dificuldade no estudo de neurorradiologia. Os dois questionamentos seguintes versaram sobre a importância da integração entre radiologia e neuroanatomia, bem como com a prática de neurologia e quanto ao estudo da disciplina. A quinta pergunta avaliou se o volume de conteúdo para pouco tempo disponível poderia ser visto como uma dificuldade no estudo de neurorradiologia.

A maioria dos alunos referiu como dificuldade para a compreensão de neurorradiologia a necessidade de ter conhecimentos básicos prévios de radiologia $(80,1 \%)$, de conhecer a neuroanatomia $(77,5 \%)$ e de correlacionar radiologia e neuroanatomia (70,9\%). Quando comparados os grupos do $2^{\circ}$ semestre e do $7^{\circ}$ semestre, houve uma tendência maior a apontar a ausência de conhecimento prático de neurologia pelos alunos do $2^{\circ}$ semestre como um fator de maior dificuldade para o aprendizado de neurorradiologia (82,6\% versus $67,4 \%$, com $\mathrm{p}<0,0018)$. Essas perguntas objetivaram avaliar se o próprio aluno tinha a capacidade de identificar o papel da integração interdisciplinar na sua formação. Além disso, a maioria dos alunos $(89,9 \%)$ aponta como fator de dificuldade para o aprendizado de neurorradiologia o grande volume de conteúdo para o pouco tempo disponível dentro da matriz curricular.

A seguir, foram realizadas quatro perguntas para avaliar quais metodologias de ensino seriam preferíveis, na opinião dos alunos, para o aprendizado da neurorradiologia: PBL (problem based learning), TBL (team based learning), aula expositiva (metodologia passiva) e disposição dos exames de neurorradiologia numa workstation de laudos.

Quando questionados se consideravam o PBL e o TBL boas metodologias para o aprendizado em neurorradiologia, os alunos do $2^{\circ}$ semestre tiveram uma maior tendência a responder positivamente em comparação com os alunos do $7^{\circ}$ semestre $(p<0,004)$, sendo que $51,7 \%$ dos alunos do 70 semestre responderam que não consideravam estes métodos adequados para o aprendizado de neurorradiologia. Um total de $78,2 \%$ dos alunos responderam que a aula expositiva seria uma boa metodologia para o aprendizado da disciplina, porém não houve diferença significativa entre os semestres. Ademais, a maioria dos alunos refere que o uso de workstations com exames reais $(91,1 \%)$ seria útil para o aprendizado.

As duas perguntas seguintes objetivaram compreender se um e-book e um aplicativo direcionados para a graduação poderiam ser úteis no aprendizado de neurorradiologia. Quando perguntados sobre a criação de um e-book, $85,6 \%$ dos alunos responderam afirmativamente, concordando que poderia auxiliar no estudo da disciplina. No caso da criação de um aplicativo, 92,3\% concordaram com um esperado efeito benéfico.

Por fim, o último item buscou avaliar de forma mais incisiva se a correlação mais explícita com a prática médica poderia ser útil no aprendizado de neurorradiologia. Um total de $98,3 \%$ dos alunos responderam que essa correlação é um conhecimento útil e necessário.

\section{DISCUSSÃO}

A vinculação entre dificuldade de compreensão de neurorradiologia e a necessidade de ter conhecimentos básicos prévios de radiologia, de neuroanatomia e de correlacionar radiologia e neuroanatomia corroboram com a necessidade de integração entre esses conteúdos. Tais percepções fortalecem a introdução da radiologia na matriz curricular desde o início do curso, de forma integrada com a anatomia ${ }^{16,10,17-23}$, como também sua manutenção nos ciclos clínicos, em conjunto com a neurologia15,19,24-26.

Outro ponto crítico destes dados é a carência no conhecimento das bases radiológicas, algo que deve ser encarado com preocupação. Na prática, percebe-se que muito comumente a matriz curricular das escolas médicas não possui módulos específicos para essa abordagem, deixando um déficit importante de conhecimento para o aluno, o qual talvez seja sanado no internato, ou apenas após a formatura, na prática médica, limitando o aprendizado de vários assuntos ao longo da graduação por falta de conhecimento sobre terminologia radiológica e princípios físicos.

Além disso, a neurorradiologia pode ser encarada como um pilar de ensino de metodologia ativa para o aprendizado de neuroanatomia. Além das dificuldades técnicas em conseguir cadáveres $^{27}$ e dos custos de uma mesa de anatomia com recursos tridimensionais, é possível identificar nos exames de ressonância magnética e de tomografia computadorizada a anatomia, a fisiologia e a patologia in vivo ${ }^{19,28}$. Uma prática já realizada em algumas universidades consiste na utilização de listas de trabalhos de casos clínicos anonimizados, previamente selecionados, onde todos os alunos podem ver as imagens sequenciais em "workstations" próprias para laudos. Além disso, a disponibilização de atlas online aproxima o aluno da prática, sem a necessidade de estar dentro do laboratório de anatomia. É certo que a visualização das imagens permite a aplicação prática de conceitos teóricos multidisciplinares ${ }^{29}$, sendo um verdadeiro tratamento para a "neurofobia"8,9,13,19.

Quando comparados os grupos do $2^{\circ}$ semestre e do $7^{\circ}$ semestre, houve uma tendência maior a apontar a ausência de conhecimento prático de neurologia pelos alunos do $2^{\circ}$ semestre como um fator de maior dificuldade para o 
aprendizado de neurorradiologia. Tal situação é apontada na literatura como um fator limitante para a fixação do conteúdo. Uma solução para essa questão é justamente introduzir alguns pontos da clínica neurológica de maneira associada, evitando que ocorra falta de interesse pelo tema por não haver conexão com aplicações práticas ${ }^{8,9,13}$.

“Um ponto que merece ser ressaltado é a escolha pormenorizada do conteúdo fundamental de neurorradiologia e neurologia para a graduação, algo que merece ser discutido dentro e fora das universidades, já que a introdução destes assuntos já no ciclo básico deve ser estimulada para uma maior fixação deste conteúdo ${ }^{8,9}$. Reuniões periódicas sobre a análise curricular contemplando coordenadores, docentes e discentes de diferentes anos na graduação poderia trazer uma rica discussão sobre como proceder na construção de conteúdo contextualizado. Também nesse sentido, a aproximação dos alunos com a instituição e entre os próprios professores permite uma maior integração do conteúdo médico, tanto horizontal quanto vertical, facilitando o aprendizado das neurociências. Merece destaque a opinião dos recém-formados atuantes em pronto-socorro e nas unidades básicas de saúde, que podem trazer importantes contribuições para a reestruturação de um plano curricular mais prático e coerente dentro das universidades. Embora seja bastante desafiador manter proximidade com ex-alunos, acredita-se que os benefícios da auto avaliação curricular universitária seriam vastos.

\section{Você atribui como uma dificuldade no estudo da graduação de neurorradiologia, a falta de correlação com a prática em neurologia?}

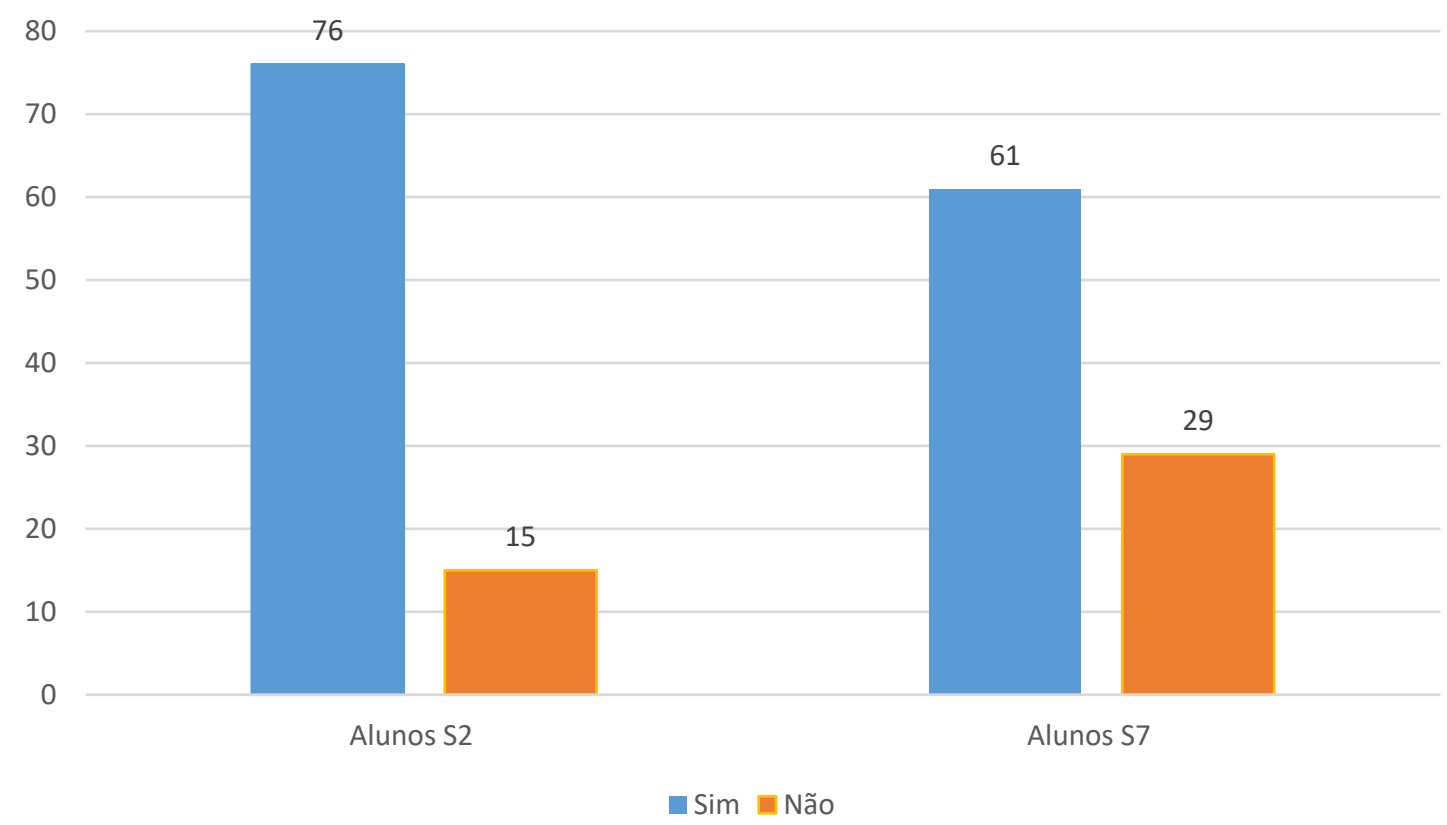

Gráfico 1.Existe correlação entre a dificuldade no estudo na graduação de neurorradiologia e a falta de correlação com a prática clínica da neurologia.

Amaiortendênciadosalunosdo $2^{\circ}$ semestrenavalorização dos métodos PBL e TBL do que os do $7 \circ$ semestre trouxe à tona uma questão enfrentada no ambiente de aula. Percebe-se, na prática docente, que a motivação com as metodologias ativas pode diminui ao longo dos anos, provavelmente devido à fadiga e à repetição, assim como ao grande volume de assunto a ser estudado e, no caso da neurorradiologia, à carência de material didático direcionado para este público ${ }^{2}$. Dessa forma, o aluno nos semestres mais avançados tem uma tendência a preferir que o conteúdo seja mais enxuto, poupando-o do estresse de procurar o conhecimento de forma livre na literatura. Nesse momento, cabe à universidade possibilitar que os alunos se sintam mais motivados na busca do conhecimento, fazendo do ambiente aula um momento inovador e evitando repetição de estratégias de ensino ao longo dos anos. $O$ incentivo ao lúdico e inclusive o próprio uso de workstations com exames reais podem ajudar nesse processo, como confirmado pela maioria dos alunos. É importante perceber quando a mudança 
de uma dinâmica é necessária, já que são bem estabelecidas as vantagens em estimular o empoderamento dos protagonistas do aprendizado, compreendendo as limitações pedagógicas dos métodos em certos momentos ${ }^{7}$.

\section{Você considera o TBL (team based learning) e o PBL (problem based learning) boas metodologias para 0 aprendizado da neurorradiologia?}

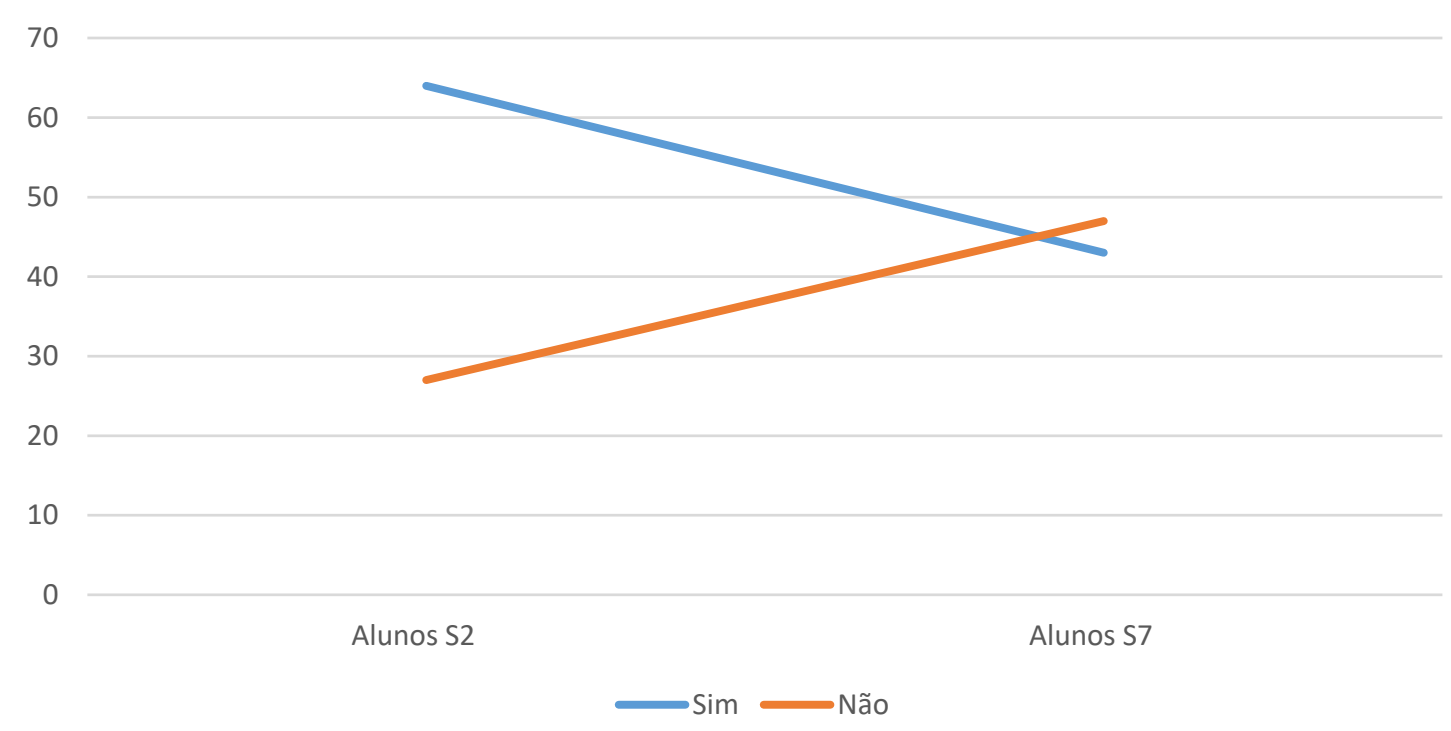

Gráfico 2.O TBL e o PBL podem ser boas metodologias para aprendizado de neurorradiologia

Uma importante queixa da maioria dos alunos foi o grande volume de conteúdo de neurorradiologia para o pouco tempo disponível dentro da matriz curricular. Percebe-se na prática que os alunos seguem por grande parte da graduação sem conhecimento adequado sobre os princípios físicos, os riscos de cada método, a terminologia dos diferentes exames e a aplicação prática de sua solicitação, algo que traria impacto positivo no decorrer do curso médico se fosse adequadamente introduzido ainda no ciclo básico².

Também existiu interesse no estudo com um e-book direcionado para a graduação e/ou uso de aplicativos confiáveis, sugerindo uma carência de material radiológico direcionado para esse público. $\mathrm{O}$ aluno do início do curso ainda não se familiarizou com vários termos médicos e a maioria tem dificuldade nas visualizações das imagens em diferentes planos. Tais habilidades podem ser melhoradas com o treinamento tridimensional, mais facilmente disponível nessas ferramentas.

Esse resultado permite considerar o uso de aplicativos e e-books como boas metodologias ativas. Tais dispositivos são altamente disponíveis, estando vinculados a smartphones e tablets, permitindo consultas rápidas e estudos mais demorados, além de possibilitar a visualização tridimensional das estruturas radiológicas, algo considerado muito importante no processo pedagógico do aprendizado em radiologia há muitos anos ${ }^{30}$. Uma estratégia bem avaliada em instituições médicas europeias para o ensino de Radiologia é o e-Learning. Trata-se justamente do uso de material eletrônico via internet para otimizar o entendimento, melhorar habilidades e promover mais experiência prática entre os alunos ${ }^{31}$.

Acredita-se que esses dados possam forçosamente ser estendidos para uma interpretação mais ampla sobre integrações efetivas e possíveis dentro de cada instituição. Entende-se que trazer o quotidiano do radiologista para a universidade, com o uso de "workstations", programas reais de laudo com armazenamento de casos anonimizados em listas de trabalho, possa despertar maior interesse nos alunos a respeito da neurorradiologia, e consequentemente no estudo das neurociênciais.

Quanto à correlação entre a neurorradiologia e a prática médica, foi praticamente unânime que é um conhecimento útil e necessário. Tal percepção é clara na literatura, uma vez que a radiologia e a clínica são indissociáveis ${ }^{8,25,26,32-34}$ e uma mesma imagem pode representar doenças completamente diferentes. Essa dependência deve servir de estímulo para a introdução precoce de casos clínicos nos semestres iniciais, estimulando o estudo multidisciplinar tanto horizontal, como vertical entre os semestres. 


\section{Você acha que a correlação mais explícita com a prática médica pode ser útil no seu aprendizado de neurorradiologia?}

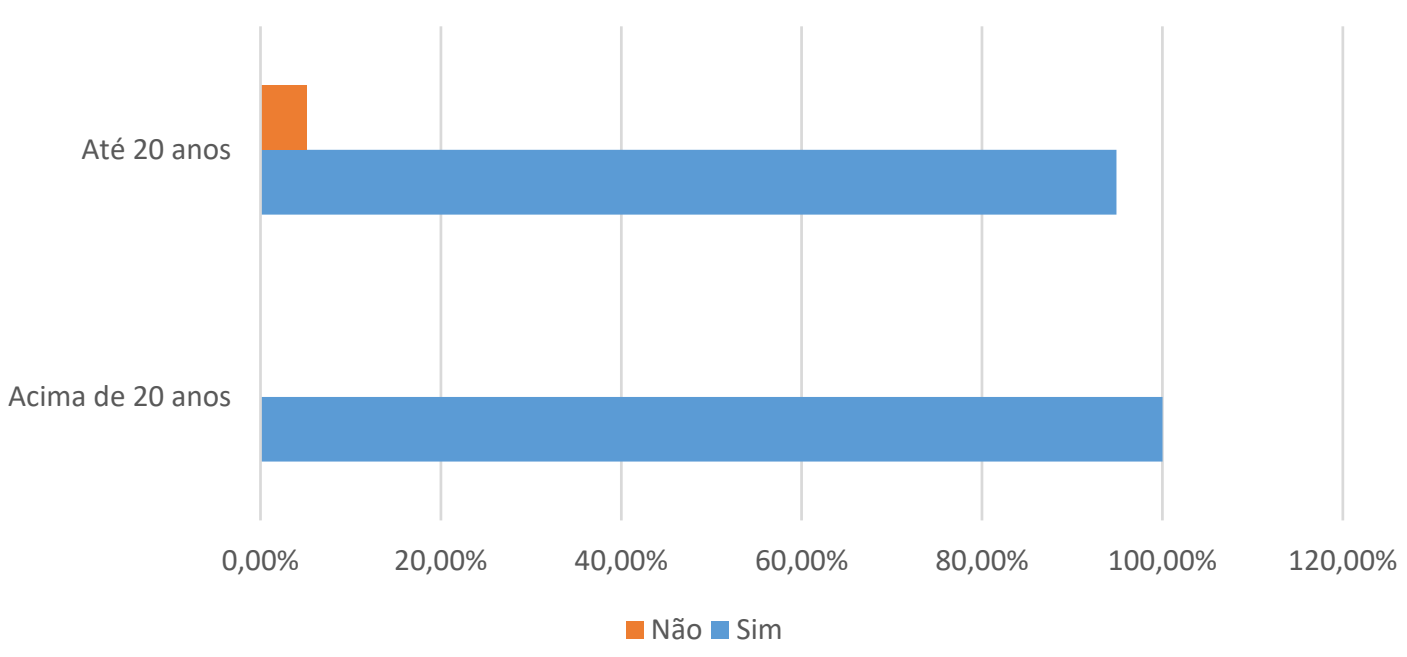

Gráfico 3. A correlação mais explicita com a prática médica pode ser útil no aprendizado de neurorradiologia.

\section{CONCLUSÕES}

Percebe-se a necessidade de uma carga horária adequada de Radiologia nos laboratórios morfofuncionais nas novas matrizes curriculares, permitindo que conceitos básicos sejam trabalhados de forma mais perene, uma vez que, na opinião dos alunos, uma limitação do aprendizado de neurorradiologia é justamente a falta de conhecimentos básicos anatômico-radiológicos. É fundamental lembrar o papel interdisciplinare multidisciplinar que a neurorradiologia desempenha perante os demais conteúdos básicos, agregando conhecimentos básicos anatômicos e clínicos já no início do ensino médico ${ }^{17,22}$. Cabe às instituições de ensino explorar as reais possibilidades de integração, tanto horizontal quanto vertical, para o melhor aprendizado das neurociências. Reuniões periódicas entre os discentes e docentes dos diferentes anos e a aproximação entre a universidade e o recém-formado podem contribuir para uma reestruturação curricular e como também para a escolha adequada dos conteúdos fundamentais a serem abordados ao longo do curso.

A neurorradiologia pode ser encarada como um pilar de ensino de metodologia ativa para o aprendizado de neuroanatomia, tanto pelas dificuldades técnicas para a aquisição de cadáveres dentro das universidades, como também pelos custos de uma mesa digital de anatomia com recursos tridimensionais ${ }^{16,22,35,36}$. É possível identificar em ressonâncias magnéticas e em tomografias computadorizadas a anatomia, a fisiologia e a patologia in vivo, permitindo inclusive planejamentos pré-cirúrgicos ${ }^{19,28}$, levando o aluno a integrar conceitos teóricos ${ }^{29}$ na prática médica, ${ }^{8,9,13,19}$.

Faz-se necessária a introdução de alguns aspectos clínicos neurológicos e de neurociências durante o ensino de neurorradiologia para que os alunos de ciclo básico compreendam a importância do que lhes é apresentado. Outro ponto igualmente fundamental é a correlação de aspectos anatômicos durante o ensino de neurologia. Essas duas estratégias são verdadeiros pilares para o tratamento e a prevenção da "neurofobia"8,9,21,32,33,36.

Na opinião dos estudantes, os conhecimentos prévios de neuroanatomia e de neurologia clínica são importantes para o aprendizado de neurorradiologia, sinalizando que a integração multidisciplinar é percebida como uma ferramenta fundamental tanto para sedimentação do conteúdo, como também para despertar o interesse em assuntos menos atrativos.

A elaboração de materiais direcionados a graduação, como e-book ou aplicativo com foco em integrar o ensino das neurociências é considerada uma boa alternativa para facilitar a compreensão da neurorradiologia, algo que pode reduzir a resistência dos estudantes ao tema, consequentemente reduzindo a neurofobia. Tal resultado permite a extensão de que quanto mais real o quotidiano do radiologista for trazido para a sala de aula, com uso de listas de exames completos, visualização das imagens em "workstations", mais interessantes os conteúdos de neurorradiologia e ciências correlatas se tornarão.

Este estudo foi realizado em apenas uma instituição, o que pode limitar generalizações. 


\section{CONTRIBUIÇÃO DOS AUTORES}

Esther de Alencar Araripe Falcão Feitosa colaborou na análise da discussão e na conclusão. Luiz Henrique Costa Neto elaborou e aplicou os questionários e participou da revisão bibliográfica. Carina de Oliveira Gregório e Letícia Nobre Limas aplicaram os questionários e participaram da revisão bibliográfica. Paulo Goberlânio de Barros Silva realizou a análise estatística. Carlos Eduardo Barros Jucá colaborou na análise da discussão e na conclusão.

\section{CONFLITO DE INTERESSES}

Os autores declaram não haver conflito de interesses neste estudo.

\section{FINANCIAMENTO}

Declaramos que não houve financiamento para a realização desta pesquisa.

\section{REFERÊNCIAS}

1. Phillips AW, Eason H, Straus CM. Student and recent graduate perspectives on radiological imaging instruction during basic anatomy courses. Anat Sci Educ. 2018;11(1):25-31.

2. Silva AF, Domingues RJS, Kietzer KS, Freitas JJS. Percepção do Estudante de Medicina sobre a inserção da radiologia no ensino de graduação com uso de metodologias ativas. Rev Bras Educ Med. 2019;43(2):95-105.

3. Kraft M, Sayfie A, Klein K, Gruppen L, Quint L. Introducing first-year medical students to radiology: implementation and impact. Acad Radiol. 2018;25(6):780-8 [acesso em 17 maio 2019]. Disponível em: http://www. ncbi.nlm.nih.gov/pubmed/30691773.

4. Chang BJ. Problem-based learning in medical school: a student's perspective. Ann Med Surg. 2016;12:88-9 [acesso em maio 2018]. Disponível em: http://www.ncbi.nlm.nih.gov/pubmed/27942381.

5. Whitehouse GH, Scarrow GD, Evans AF. Initial experiences with a formalised teaching programme in diagnostic radiology during the clinical undergraduate course. Clin Radiol. 1979 Mar;30(2):233-7.

6. Tshibwabwa E, Mallin R, Fraser M, Tshibwabwa M, Sanii R, Rice J, et al. An integrated interactive-spaced education radiology curriculum for preclinical students. J Clin Imaging Sci. 2017;7:22.

7. Gomes AP, Rego S. Transformação da educação médica: é possível formar um novo médico a partir de mudanças no método de ensinoaprendizagem? Rev Bras Educ Med. 2011;35(4):557-66.

8. Ridsdale L, Massey R, Clark L. Preventing neurophobia in medical students, and so future doctors. Pract Neurol. 2007 Apr;7(2):116-23.

9. Shiels L, Majmundar P, Zywot A, Sobotka J, Lau CSM, Jalonen TO. Medical student attitudes and educational interventions to prevent neurophobia: a longitudinal study. BMC Med Educ. 2017;17(1)225 [acesso em 17 maio 2019]. Disponível em: https://bmcmededuc.biomedcentral.com/track/ pdf/10.1186/s12909-017-1055-4.

10. Heptonstall NB, Ali T, Mankad K. Integrating radiology and anatomy teaching in medical education in the uk-the evidence, current trends, and future scope. Acad Radiol. 2016 Apr;23(4):521-6.

11. Cyrino EG, Toralles-Pereira ML. Trabalhando com estratégias de ensinoaprendizado por descoberta na área da saúde: a problematização e a aprendizagem baseada em problemas. Cad Saude Publica. 2004;20(3):7808 [acesso em 29 jun 2018]. Disponível em: http://www.scielo.br/scielo. php?script=sci_arttext\&pid=S0102-311X2004000300015\&lng=pt\&tlng=pt.

12. Moraes MAA, Manzini EJ. Concepções sobre a aprendizagem baseada em problemas: um estudo de caso na Famema. Rev Bras Educ Med. 2006;30(3):125-35.

13. Jozefowicz RF. Neurophobia: the fear of neurology among medical students. Arch Neurol. 1994;51:328-9.

14. Farias PAM, Martin ALAR, Cristo CS. Aprendizagem ativa na educação em saúde: percurso histórico e aplicações. Rev Bras Educ Med. 2015:39(1):143-50.
15. Ekelund L, Elzubeir M. Diagnostic radiology in an integrated curriculum: evaluation of student appraisal. Acad Radiol. 2000 Nov;7(11):965-70 [acesso em 27 maio 2019]. Disponível em: http://www.ncbi.nlm.nih.gov/ pubmed/11089699.

16. McLachlan JC, Bligh J, Bradley P, Searle J. Teaching anatomy without cadavers. Med Educ J. 2004;38:418-24.

17. Schober A, Pieper CC, Schmidt R, Wittkowski W. Anatomy and imaging: 10 years of experience with an interdisciplinary teaching project in preclinical medical education - from an elective to a curricular course. RoFo Fortschritte auf dem Gebiet der Rontgenstrahlen und der Bildgeb Verfahren. 2014;186(5):458-65.

18. Murphy KP, Crush L, O'Malley E, Daly FE, Twomey M, O'Tuathaigh CMP. Medical student perceptions of radiology use in anatomy teaching. Anat Sci Educ. 2015;8:510-7.

19. Gunderman RB, Siddiqui AR, Heitkamp DE, Kipfer HD. The vital role of radiology in the medical school curriculum. AJR Am J Roentgenol. 2003;180(5):1239-42.

20. Arantes M, Arantes J, Ferreira MA. Tools and resources for neuroanatomy education: a systematic review. BMC Med Educ. 2018 May 3;18(1):94.

21. avaid MA, Chakraborty S, Cryan JF, Schellekens H, Toulouse A. Understanding neurophobia: reasons behind impaired understanding and learning of neuroanatomy in cross-disciplinary healthcare students. Anat Sci Educ. 2018;11(1):81-93.

22. Sheikh AH, Barry DS, Gutierrez H, Cryan JF, O'Keeffe GW. Cadaveric anatomy in the future of medical education: What is the surgeons view? Anat Sci Educ. 2016 Mar 1;9(2):203-8.

23. Retrouvey M, Trace AP, Goodmurphy CW, Shaves S. Redefining the radiology curriculum in medical school: vertical integration and global accessibility. Am J Roentgenol. 2018;210(1):118-22.

24. Brich J, Jost M, Brüstle P, Giesler M, Rijntjes M. Teaching neurology to medical students with a simplified version of team-based learning. Neurology. 2017 Aug 8;89(6):616-22. doi: 10.1212/WNL.0000000000004211.

25. Jones M, Gardner M. Neurophobia - can we overcome it to teach neurology in primary care? Proceedings of the 45th Annual Meeting of the Society of Academic Primary Care (SAPC); 2016. Society of Academic Primary Care (SAPC): Dublin, Ireland.

26. Charalambous N, Goh A, Los F, Sharma K. Does understanding basic neuroscience cure neurophobia? J Neurol Neurosurg Psychiatry. 2015;86(11):e4.140-e4.

27. Eppler E, Serowy S, Link K, Filgueira L. Experience from an optional dissection course in a clinically-orientated concept to complement system-based anatomy in a reformed curriculum. Anat Sci Educ. 2018 Jan;11(1):32-43 [acesso em 23 maio 2018]. Disponível em: http://www. ncbi.nlm.nih.gov/pubmed/28608954.

28. Familiari G, Relucenti M, Heyn R, Baldini R, D'Andrea G, Familiari P, et al The value of neurosurgical and intraoperative magnetic resonance imaging and diffusion tensor imaging tractography in clinically integrated neuroanatomy modules: a cross-sectional study. Anat Sci Educ. 2013 Sep;6(5):294-306 [acesso em 23 maio 2018]. Disponível em: http://www. ncbi.nlm.nih.gov/pubmed/23509097.

29. Groth M, Barthe KG, Riemer M, Ernst M, Herrmann J, Fiehler J, et al. Critical analysis of an e-learning and interactive teaching module with respect to the interpretation of emergency computed tomography of the brain. Fortschr Röntgenstr. 2018;190:334-40.

30. Tuddenham WJ. Computers in radiology. Appl Radiol. 1975;4(6):57-62.

31. Zafar S, Safdar S, Zafar AN. Evaluation of use of e-learning in undergraduate radiology education: a review. Eur J Radiol. 2014;83:2277-87.

32. Hudson JN. Linking neuroscience theory to practice to help overcome student fear of neurology. Med Teach. 2006; Nov;28(7):651-3.

33. Zambrano DM, Vásquez RS. Neurofobia entre los estudiantes de la carrera de Medicina de sexto a décimo semestre en la Universidad Católica Santiago de Guayaquil. Rev Ecuat Neurol. 2013;22:1-3.

34. Tarolli CG, Józefowicz RF. Managing neurophobia: how can we meet the current and future needs of our students? Semin Neurol. 2018;38(4):407-12.

35. Turney BW. Anatomy in a modern medical curriculum. Ann R Coll Surg Engl. 2007 Feb;89(2):104-7.

36. Ekstrand C, Jamal A, Nguyen R, Kudryk A, Mann J, Mendez I. Immersive and interactive virtual reality to improve learning and retention of neuroanatomy in medical students: a randomized controlled study. $C$ Open. 2018;6(1):103-9. 